\title{
STACKED POINTNETS FOR ALIGNMENT OF PARTICLES WITH CYLINDRICAL SYMMETRY IN SINGLE MOLECULE LOCALIZATION MICROSCOPY
}

\author{
Youbo Fan ${ }^{1}$, Sylvain Faisan ${ }^{1}$, Étienne Baudrier ${ }^{1}$, Fabian Zwettler $^{2}$, Markus Sauer $^{2}$, Denis Fortun ${ }^{1}$ \\ ${ }^{1}$ ICube UMR 7357, Université de Strasbourg, CNRS, Strasbourg, France \\ ${ }^{2}$ Lehrstuhl für Biotechnologie und Biophysik, Biozentrum, Universität Würzburg, Germany
}

\begin{abstract}
Single molecule localization microscopy is an essential observation tool in biology that yields data in the form of point clouds. It is still limited by an anisotropic resolution and inhomogeneous labeling density. This issue can be addressed by reconstructing a single model from multiple aligned copies of the same particle. However, generic registration methods fail to align point clouds in the presence of anisotropic noise and outliers. Therefore, we propose an alignment method dedicated to a common type of particle geometry, namely cylindrical symmetry. We focus on the centriole, a fundamental macromolecular assembly with ninefold cylindrical symmetry. We design a neural network based on stacked PointNet architectures that estimates the center and axis of symmetry of individual particles in SMLM, in order to align them in the same canonical space. We demonstrate the robustness of our approach on simulated and real dSTORM data.
\end{abstract}

Index Terms - SMLM, centriole, point clouds, neural networks, PointNet

\section{INTRODUCTION}

Single molecule localization microscopy (SMLM) is one of the most widely used techniques of superresolution fluorescence microscopy [1]. The principle is to acquire images populated with a few isolated fluorophores, such that their position can be determined accurately with a localization algorithm [2]. The resulting data takes the form of a point cloud, where each point represents a fluorophore and is associated with an uncertainty on its localization. SMLM methods are able to reach near nanometric resolution in $2 \mathrm{D}$, and thus give access to macromolecular assemblies smaller than the diffraction limit. However, the 3D resolution is usually 3 to 5 times lower in the axial direction and prevents from accurate 3D observation, as illustrated in Fig.1. Moreover, the fluorescent labelling does not cover homogeneously the surface of the centriole, which can yield large unlabelled regions and a significant amount of outliers.

To circumvent these issues, we opt for the single particle reconstruction paradigm: instead of observing a unique centriole, multiple copies of the same rigid particle are im-

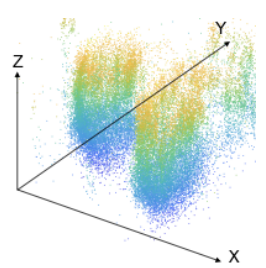

(a)

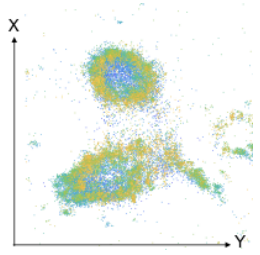

(b)

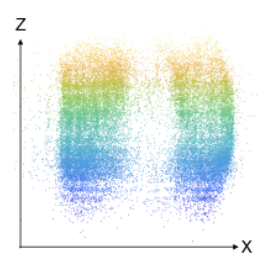

(c)
Fig. 1. Example of point cloud acquired in SMLM (with dSTORM [6]). (a) 3D view; (b) XY plane with high resolution; (c) XZ plane with a low resolution in the $\mathrm{Z}$-direction.

aged with random orientations, and a single average model is estimated. The combination of different orientations and labellings yields an improvement of the 3D resolution. This approach has been investigated in a few works in 2D SMLM $[3,4]$, and recently in 3D [5] but only in a simplified setting where the orientation angles between particles are small. Single particle reconstruction involves two tasks: alignment of the particles in the same pose and reconstruction of the particle model. In this work, we address the alignment problem.

Particle alignment in SMLM amounts to multiview point clouds registration, which has been a longstanding research topic in computer vision. The standard framework consists in computing registration between all pairs of point clouds and to apply motion synchronization techniques [7]. We applied state-of-the-art pairwise registration methods based on deep neural networks $[8,9]$ to our SMLM data, but we observed that they fail to produce satisfying results. The main reason is that the local feature computation involved in $[8,9]$ is designed for relatively clean data with a small amount of noise. In SMLM, the high level of anisotropic noise drastically alters the shapes of differently oriented centriole, as it can be seen by comparing Fig.2(c) and Fig.2(d), such that matching local features becomes a difficult task.

In this paper, we circumvent this issue by addressing the alignment problem from a different perspective. Instead of developing a generic registration method, we leverage prior knowledge about the geometry of the particle. We focus here on the centriole, a macromolecular assembly involved in several fundamental cellular processes [10, 11], which has attracted a lot of research to understand its role in diseases such 
as cancer or ciliopathies [12]. The structure of the centriole has a ninefold cylindrical symmetry. It implies that its orientation is mostly determined by its axis of symmetry, which corresponds to the first two Euler angles. The variation of the third Euler angle is restricted to the range $[0,2 \pi / 9]$. Therefore, we can get a coarse alignment of the centrioles by estimating their axis and center of mass. This is a first necessary step towards further refinement and reconstruction that we let for future work. Our method is based on a four-stage neural network architecture that builds upon the PointNet global features [13]. To create realistic training data, we use results that revealed the ultrastructure of the centriole [14]. We validate the relevance and accuracy of each stage of our approach on synthetic point clouds, and on real dSTORM data [6].

\section{CENTRIOLE STRUCTURE AND SIMULATION}

We focus on data with fluorescent labelling of tubulin, which is one of the proteins that compose the centriole and is located in its outer wall. Figure 2(a) shows our simulated model of the ninefold cylindrical symmetry structure of tubulin, based on the analysis reported in [14]. It is composed of nine microtubule triplets organized symmetrically on a barrel-like structure. The direction is identified by a larger radius at the bottom of the barrel, and a smaller radius at the top (see [14] for details). The red arrow in Fig.2(a) indicates the axis and direction of the centriole. In what follows, we call axis the undirected axis of symmetry and direction the binary value that indicates the top of the centriole on this axis. In our simulations, we randomly sample the parameters of the model to reflect the natural variability of the centriole.

After rotating a noise-free centriole, our SMLM simulation process begins by adding typical non-centriolar structures that we considered as outliers, shown in Fig.2(b). We model three classes of outliers: microtubules represented as filaments, Gaussian clusters of points and scattered points sampled from a uniform law. We also account for the inhomogeneity of the fluorescent labelling by removing groups of points in the centriole structure. Then, we introduce anisotropic localization uncertainty (Fig.2(c)) to obtain the final simulated data. To create realistic noise, we sampled from estimated distributions of the lateral and axial uncertainties from real dSTORM data.

Figure 2(d) shows a simulated centriole tilted by 90 degrees with respect to (c). It illustrates the effect of the anisotropic uncertainty: the noise on the lateral axis prevails on the cylindrical shape, such that the barrel is filled with noise in Fig.2(d) and registration of (d) on (c) fails with standard methods.

\section{NEURAL NETWORKS FOR POSE ESTIMATION}

Our purpose is to estimate the axis, direction and center of mass of centrioles represented by point clouds. The sim-

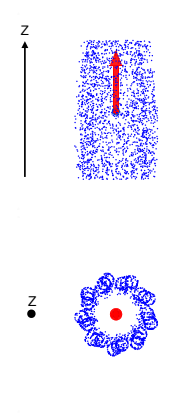

(a)

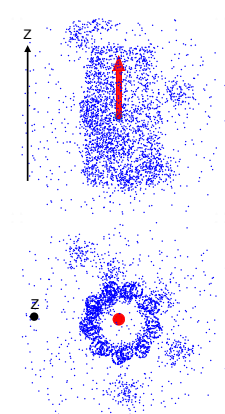

(b)

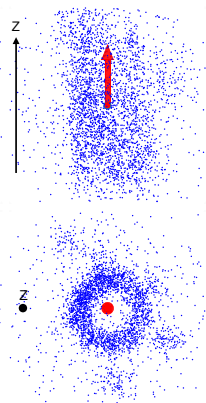

(c)

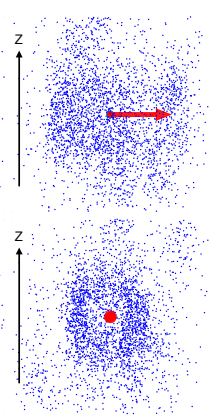

(d)
Fig. 2. Simulation of centriole in SMLM. The red arrow represents the axis and the direction. The top line shows side views of the centriole, and the bottom line shows views orthogonal to the axis of symmetry. Columns (a), (b) and (c) show the three steps of the simulation process: (a) simulated centriole model [14], (b) add outliers and missing parts, (c) add anisotropic uncertainties. Column (d) shows a simulated centriole tilted by 90 degrees with respect to (c).

plest approach would be to use a regression network to directly estimate the parameters of the axis and center from point cloud features such as PointNet [13]. This principle has been applied for estimating canonical spatial transformation [13], hand pose [15] or bounding boxes [16]. However, this approach yields unsatisfactory results in our case due to the aforementioned noise and partial labelling problems. Therefore, we designed a sequential pipeline, summarized in Fig.3, that divides the original task into four sub-problems solved using neural networks (NN1 to NN4). The four steps are as follows:

- NN1 performs a segmentation of the centriole from the original noisy point cloud $P C_{1} . P C_{2}$ is obtained from $P C_{1}$ by removing points detected as outliers.

- NN2 estimates the center of mass $\mathbf{c}$ of the centriole from $\mathrm{PC}_{2} . \mathrm{PC}_{3}$ is obtained by translating $\mathrm{PC}_{2}$ so that the centriole is centered at $(0,0,0)$.

- NN3 estimates a unit vector $\mathbf{v}$ aligned with the axis of the centriole from $P_{3} . P C_{4}$ is then obtained by rotating $P_{3}$ to align the axis of the centriole with the $z$-axis.

- NN4 estimates a binary variable that indicates the direction of the centriole.

The networks NN1 to NN4 are all composed of two modules: the first one computes a feature vector encoding global shape information, and the second module operates on the feature vector and is adapted to each specific task.

First module The feature vector is computed with the same PointNet architecture in the four networks [13]. The interest of PointNet for our problem is that it encodes global shape information. Indeed, since the local geometry is distorted by the presence of anisotropic noise and undesirable structures, local features are not able not capture the characteristic shape of centrioles. PointNet performs independent pointwise feature computation followed by a symmetric pooling function to 


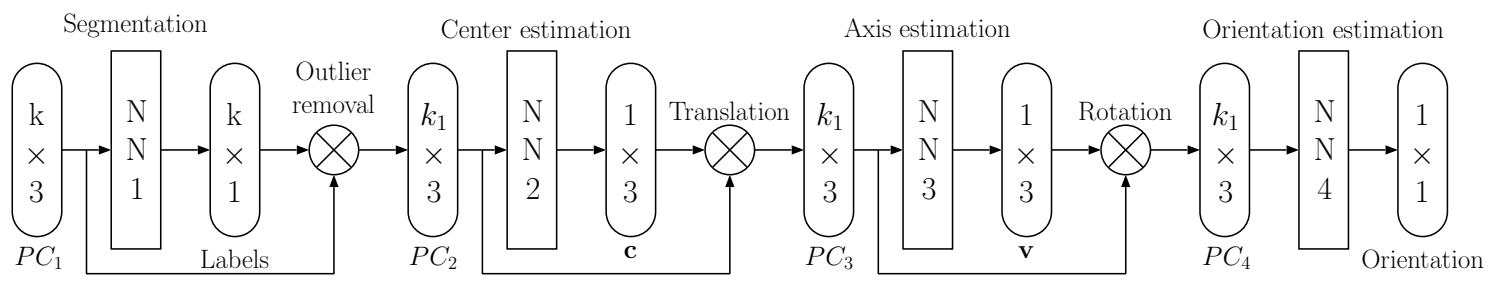

Fig. 3. Pipeline of the proposed method.

achieve invariance to permutation in unordered point clouds. We used average pooling, which we found to be more robust to noise than the usual max-pooling. The original PointNet architecture contains two transformation networks (T-nets) that estimate an affine transformation to achieve pose invariant feature computation. As mentioned at the beginning of the Section, this pose estimation module does not work in our case and has been removed.

Second module For NN1, we used the segmentation module of [13]. For NN2 and NN3, we defined fully connected regression networks adapted to the output size expected for each stage (see Fig.3). Finally, for NN4 we used a binary classification network also described in [13].

The overall architecture can be seen as stacked PointNet regression and classification networks that successively accomplish one step of the final goal. Each subtask is necessary and benefits from the estimation of previous step, as will be demonstrated experimentally in Section 4.

We train each network separately with different loss functions adapted to each task. For the segmentation task of NN1 we used the mean absolute error, which yielded better results than binary cross entropy as reported in [17]. For the regression task NN2, we used the Mean Square Error $(M S E)$ $L_{\mathbf{c}}=\frac{1}{N} \sum_{i=1}^{N} L_{\mathbf{c}, i}$ where $\mathrm{N}$ is the number of point clouds,

$$
L_{\mathbf{c}, i}=\left\|\mathbf{c}_{i}-\mathbf{c}_{i}^{g t}\right\|_{2}^{2},
$$

and $\mathbf{c}_{i}^{g t}, \mathbf{c}_{i}$ are the ground truth and the estimated center of the $i^{t h}$ point cloud, respectively. For NN3, we only aim at the direction of the vector $\mathbf{v}$, which means that both $\mathbf{v}$ and $-\mathbf{v}$ are valid vectors. To account for this, we define the loss function as $L_{\mathbf{v}}=\frac{1}{N} \sum_{i=1}^{N} L_{\mathbf{v}, i}$ with

$$
L_{\mathbf{v}, i}=\min \left\{\left\|\mathbf{v}_{i}^{g t}-\mathbf{v}_{i}\right\|_{2}^{2},\left\|\mathbf{v}_{i}^{g t}+\mathbf{v}_{i}\right\|_{2}^{2}\right\},
$$

where $\mathbf{v}_{i}^{g t}$ and $\mathbf{v}_{i}$ are the ground truth and the estimated vector that represent the axis of the $i^{\text {th }}$ point cloud, respectively. Note that $\left\|\mathbf{v}_{i}^{g t}-\mathbf{v}_{i}\right\|_{2}^{2}$ corresponds to $2(1-\cos (\theta))$ where $\theta$ is the angle between $\mathbf{v}_{i}^{g t}$ and $\mathbf{v}_{i}$ since both vectors are unitary. Finally, the loss of the binary classification network NN4 is defined as the binary cross entropy.

Each neural network has been trained individually and sequentially (from NN1 to NN4) using 1000 point sets as a train set, 200 as a validation set, and 800 as a test set. When training the $n$-th neural network, the parameters of the $n-1$ pre- vious neural networks are freezed. The optimization is performed with the Adam optimizer with a batch size equal to 16. The learning rate, the $\ell_{2}$ regularization weight and the number of epochs have been optimized using the validation set. The other hyperparameters of the Adam optimizer are set to the recommended values in [18].

\section{RESULTS}

\subsection{Synthetic data}

To demonstrate the necessity of each step of our pipeline, we analyze three variants of the proposed approach (that we denote $P$ ) by removing or merging different modules as follows:

- P1: The segmentation network NN1 is removed.

- P2: The center estimation network NN2 is removed and the center is computed as the center of mass of the point cloud. Note that the center of the point cloud is equivalent to the center of the centriole if there is no noise and no missing part.

- P3: The axis and direction networks NN3 and NN4 are merged. It means that NN4 is removed and NN3 outputs jointly the axis and the direction. The architecture remains unchanged and the loss function is defined similarly to (2) but with a single direction $\left(L_{\mathbf{v}, i}=\left\|\mathbf{v}_{i}^{g t}-\mathbf{v}_{i}\right\|_{2}^{2}\right)$.

Results obtained on the test dataset are shown in Table 1 for the four approaches. The first column represents the percentage of correctly segmented points. The second and third columns are the MSE for the estimation of the center $\mathbf{c}$ and the axis $\mathbf{v}((1)$ and (2)), respectively. The numbers in parentheses are the percentages of successful estimations. We consider that an estimation is successful if $L_{\mathbf{c}, i}$ is below $0.05^{2}=$ $25.10^{-4}$ (which approximately corresponds to an error of one twentieth of the size of the centriole since most of the points are in the unit cube) and $L_{\mathbf{v}, i}$ is below $0.3^{2}=900.10^{-4}$ (which approximately corresponds to an error of 17 degrees). These thresholds have been determined visually and examples are shown in Fig.4. The fourth column is the percentage of correct direction classification. The last column is the percentage of jointly correct estimations of the center, axis and direction.

We observe that as soon as one of the four modules is removed, the results are significantly deteriorated. Without the segmentation step $(P 1)$, the accuracy of all the stages is 


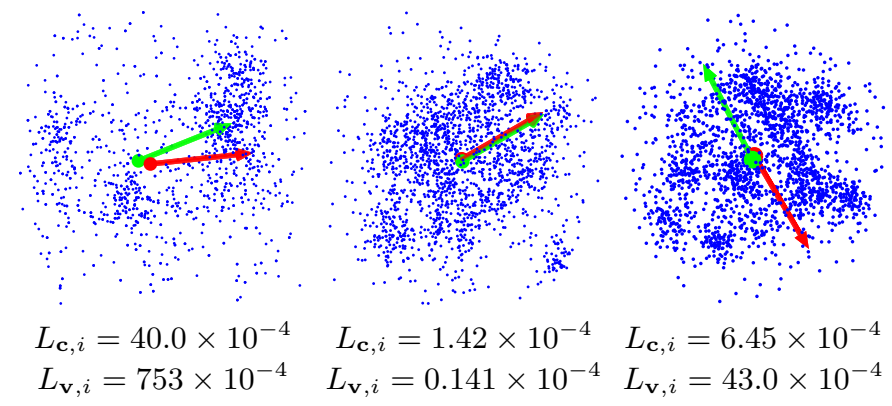

Fig. 4. Results on simulated data. The red arrow is the ground truth axis, and the green arrow is the estimation.

\begin{tabular}{|c|c|c|c|c|c|}
\hline & Seg. & $L_{\mathbf{c}} \times 10^{4}$ & $L_{\mathbf{v}} \times 10^{4}$ & Orient. & All \\
\hline$P$ & 86.1 & $\mathbf{8 . 1 0}(\mathbf{9 3 . 5})$ & $\mathbf{9 3 . 8}(99.1)$ & $\mathbf{8 3 . 4}$ & $\mathbf{7 9 . 2}$ \\
\hline$P 1$ & $\mathrm{X}$ & $15.3(80.3)$ & $621.1(89.8)$ & 71.2 & 55.8 \\
\hline$P 2$ & 86.1 & $43.3(52.9)$ & $649.7(82.5)$ & 81.0 & 40.0 \\
\hline$P 3$ & 86.1 & $\mathbf{8 . 1 0}(\mathbf{9 3 . 5})$ & $599.9(85.6)$ & 53.1 & 43.9 \\
\hline
\end{tabular}

Table 1. Results on the test dataset (see the text for details).

affected and the overall accuracy falls at $55.8 \%$. When the center estimation is removed $(P 2)$, the centriole is not properly centered and it prevents from an accurate estimation of the axis. Finally, results obtained with $P 3$ show that estimating jointly the axis and the direction is significantly less efficient than our independent sequential estimation. Also note that the direction estimation is particularly challenging since the difference of radius between the bottom and the top of the centriole is so small that it is most often impossible to identify it visually. These results demonstrate that the direct pose estimation is impracticable, and it validates our general approach of dividing the pose estimation problem into several sub-problems solved sequentially.

Figure 4 shows visual results that illustrate the quality of the estimation and the complexity of the data. It also illustrates the thresholds chosen to compute the success rates in columns three and four of Table 1.

\subsection{Real data}

We applied our method on real data acquired with dSTORM combined with expansion microscopy [19]. Each acquisition usually contains several centrioles (see Fig.1). We manually cropped a total of 42 centrioles from the original data. Figure 4.2 shows examples of estimation results on this data. Since we do not have access to the ground truth poses, we had to rely on visual inspection to evaluate the accuracy of the estimation. It is visually difficult to detect the direction, so we focused on the estimation of the axis only. By considering a similar visual threshold than the one used for the computation of the success rate in column four of Table 1 , we observed that the estimation was successful on 40 centrioles over 42 (this represents a percentage of approximately $95 \%$ ). We can
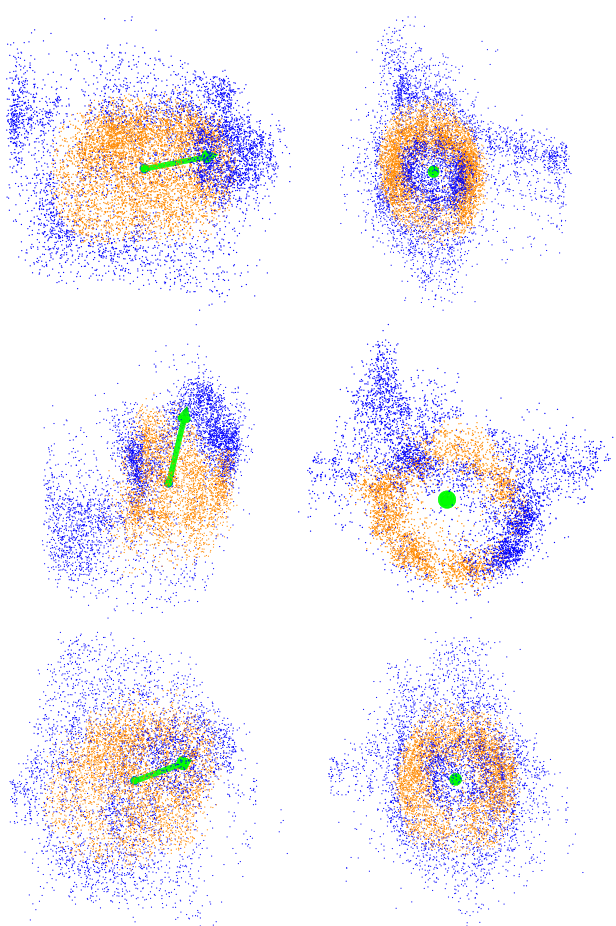

Fig. 5. Results on real dSTORM data. The green arrow is the estimated axis and direction. The segmentation step classified the orange points as centriole points and the blue points as outliers. Each row shows one centriole with two views. The left column shows a random view, and the the right column shows a view orthogonal to the estimated axis.

conclude that the model achieves good generalization properties on real data despite the fact that it has been learned on synthetic data.

\section{CONCLUSION AND FUTURE WORK}

We have presented a method for estimating the axis, direction and center of structures with cylindrical symmetry, with an application to the centriole. Our method is based on a neural network architecture that stacks PointNet feature computation with regression and classification networks. We demonstrated that our subdivision of the problem in four stages is crucial to obtain satisfying results. This work is a first step towards a full single particle reconstruction method. It provides an initialization of pose estimation that will be used for refinement and reconstruction in future work. We also plan to investigate improvements such as end-to-end training of the network, or iterative refinement. Our framework can be applied to other particles with cylindrical symmetry (e.g the nuclear pore [20]), and it could also be extended to other types of geometrical prior. 


\section{ACKNOWLEDGMENTS}

This work was supported by the French National Research Agency (ANR) through the SP-Fluo project (ANR-20-CE450007).

\section{COMPLIANCE WITH ETHICAL STANDARDS}

This study was performed in line with the principles of the Declaration of Helsinki.

\section{REFERENCES}

[1] Markus Sauer, "Localization microscopy coming of age: from concepts to biological impact," Journal of cell science, vol. 126, no. 16, pp. 3505-3513, 2013.

[2] Daniel Sage, Thanh-An Pham, Hazen Babcock, Tomas Lukes, Thomas Pengo, Jerry Chao, Ramraj Velmurugan, Alex Herbert, Anurag Agrawal, Silvia Colabrese, et al., "Superresolution fight club: assessment of $2 \mathrm{~d}$ and $3 \mathrm{~d}$ single-molecule localization microscopy software," Nature methods, vol. 16, no. 5, pp. 387-395, 2019.

[3] Anna Szymborska, Alex De Marco, Nathalie Daigle, Volker C Cordes, John AG Briggs, and Jan Ellenberg, "Nuclear pore scaffold structure analyzed by super-resolution microscopy and particle averaging," Science, vol. 341 , no. 6146, pp. 655658, 2013.

[4] Hamidreza Heydarian, Florian Schueder, Maximilian T Strauss, Ben Van Werkhoven, Mohamadreza Fazel, Keith A Lidke, Ralf Jungmann, Sjoerd Stallinga, and Bernd Rieger, "Template-free $2 \mathrm{~d}$ particle fusion in localization microscopy," Nature methods, vol. 15, no. 10, pp. 781-784, 2018.

[5] Hamidreza Heydarian, Adrian Przybylski, Florian Schueder, Ralf Jungmann, Ben van Werkhoven, Jan Keller-Findeisen, Jonas Ries, Sjoerd Stallinga, Mark Bates, and Bernd Rieger, "Three dimensional particle averaging for structural imaging of macromolecular complexes by localization microscopy," bioRxiv, p. 837575, 2019.

[6] Mike Heilemann, Sebastian Van De Linde, Mark Schüttpelz, Robert Kasper, Britta Seefeldt, Anindita Mukherjee, Philip Tinnefeld, and Markus Sauer, "Subdiffraction-resolution fluorescence imaging with conventional fluorescent probes," Angewandte Chemie International Edition, vol. 47, no. 33, pp. 6172-6176, 2008.

[7] Uttaran Bhattacharya and Venu Madhav Govindu, "Efficient and robust registration on the $3 \mathrm{~d}$ special euclidean group," in IEEE International Conference on Computer Vision, 2019, pp. 5885-5894.

[8] Yue Wang and Justin M Solomon, "Deep closest point: Learning representations for point cloud registration," in IEEE International Conference on Computer Vision, 2019, pp. 35233532.

[9] Zi Jian Yew and Gim Hee Lee, "Rpm-net: Robust point matching using learned features," in IEEE Conference on Computer Vision and Pattern Recognition, 2020, pp. 11824-11833.
[10] Michel Bornens and Juliette Azimzadeh, "Origin and evolution of the centrosome," in Eukaryotic Membranes and Cytoskeleton, pp. 119-129. Springer, 2007.

[11] Davide Gambarotto, Fabian U Zwettler, Maeva Le Guennec, Marketa Schmidt-Cernohorska, Denis Fortun, Susanne Borgers, Jörn Heine, Jan-Gero Schloetel, Matthias Reuss, Michael Unser, et al., "Imaging cellular ultrastructures using expansion microscopy (u-exm)," Nature methods, vol. 16, no. 1, pp. 71-74, 2019.

[12] Pierre Gönczy, "Centrosomes and cancer: revisiting a longstanding relationship," Nature reviews cancer, vol. 15, no. 11, pp. 639-652, 2015.

[13] Charles R Qi, Hao Su, Kaichun Mo, and Leonidas J Guibas, "Pointnet: Deep learning on point sets for 3d classification and segmentation," in IEEE Conference on Computer Vision and Pattern Recognition, 2017, pp. 652-660.

[14] Maeva Le Guennec, Nikolai Klena, Davide Gambarotto, Marine H Laporte, Anne-Marie Tassin, Hugo Van den Hoek, Philipp S Erdmann, Miroslava Schaffer, Lubomir Kovacik, Susanne Borgers, et al., "A helical inner scaffold provides a structural basis for centriole cohesion," Science advances, vol. 6, no. 7, pp. eaaz4137, 2020.

[15] Liuhao Ge, Yujun Cai, Junwu Weng, and Junsong Yuan, "Hand pointnet: 3d hand pose estimation using point sets," in IEEE conference on Computer Vision and Pattern Recognition, 2018, pp. 8417-8426.

[16] Charles R Qi, Wei Liu, Chenxia Wu, Hao Su, and Leonidas J Guibas, "Frustum pointnets for $3 \mathrm{~d}$ object detection from rgbd data," in IEEE conference on Computer Vision and Pattern Recognition, 2018, pp. 918-927.

[17] Marie-Julie Rakotosaona, Vittorio La Barbera, Paul Guerrero, Niloy J Mitra, and Maks Ovsjanikov, "Pointcleannet: Learning to denoise and remove outliers from dense point clouds," in Computer Graphics Forum. Wiley Online Library, 2020, vol. 39, pp. 185-203.

[18] Diederik P. Kingma and Jimmy Ba, "Adam: A method for stochastic optimization," 2015, International Conference for Learning Representations, San Diego, 2015.

[19] F.U. Zwettler, S. Reinhard, D. Gambarotto, T.D.M. Bell, V. Hamel, Guichard P., and M. Sauer, "Molecular resolution imaging by post-labeling expansion single-molecule localization microscopy (Ex-SMLM)," Nature Communications, vol. 11, no. 3388, 2020.

[20] Daniel H Lin, Tobias Stuwe, Sandra Schilbach, Emily J Rundlet, Thibaud Perriches, George Mobbs, Yanbin Fan, Karsten Thierbach, Ferdinand M Huber, Leslie N Collins, et al., "Architecture of the symmetric core of the nuclear pore," Science, vol. 352, no. 6283, 2016. 